\title{
Absence of Shocks in an Initially Dilute Collisionless Plasma ${ }^{\star}$
}

Robert T. Glassey ${ }^{1}$ and Walter A. Strauss ${ }^{2}$

1 Department of Mathematics, Indiana University, Bloomington, IN 47405, USA

2 Department of Mathematics, Brown University, Providence, RI 02912, USA

\begin{abstract}
The Cauchy Problem for the relativistic Vlasov-Maxwell equations is studied in three space dimensions. It is assumed that the initial data satisfy the required constraints and have compact support. If in addition the data have sufficiently small $C^{2}$ norm, then a unique $C^{1}$ solution to this system is shown to exist on all of spacetime.
\end{abstract}

\section{Introduction}

A plasma is said to be collisionless if the collisions between the particles are unimportant relative to the electromagnetic forces. Such a plasma is governed by the Vlasov-Maxwell equations. An ionized gas which is sufficiently hot, such as a powered-up fusion reactor, or sufficiently dilute, such as the solar wind, the ionosphere or a collection of galactic nebulae, is thought to be well modeled by these equations. In this paper we prove that if a plasma is sufficiently dilute initially, then it remains so for all time and no shock or other singularity can ever develop.

We postulate several $(N)$ species of particles (electrons, different types of ions, etc.) with masses $m_{\alpha}$, charges $e_{\alpha}$ and densities $f_{\alpha}(t, x, v)\left(t \geqq 0, x \in \mathbb{R}^{3}, v \in \mathbb{R}^{3}\right.$, $1 \leqq \alpha \leqq N)$. The total charge and current densities are

$$
\begin{aligned}
\rho(t, x) & =4 \pi \int_{\mathbb{R}^{3}} \sum_{\alpha} e_{\alpha} f_{\alpha} d v, \\
j(t, x) & =4 \pi \int_{\mathbb{R}^{3}} \sum_{\alpha} e_{\alpha} \hat{v}_{\alpha} f_{\alpha} d v,
\end{aligned}
$$

where, in the relativistic version we consider,

$$
\hat{v}_{\alpha} \equiv v\left[m_{\alpha}^{2}+|v|^{2} / c^{2}\right]^{-1 / 2}
$$

has the interpretation of velocity, $v$ is the momentum, and $c$ is the speed of light. Each $f_{\alpha}$ will satisfy a continuity equation (the Vlasov equation) which is coupled

* Research supported in part by NSF DMS 85-20662 and NSF DMS 84-20957 
to the Maxwell system:

$$
\partial_{t} f_{\alpha}+\hat{v}_{\alpha} \cdot \nabla_{x} f_{\alpha}+e_{\alpha}\left(E+c^{-1} \hat{v}_{\alpha} \wedge B\right) \cdot \nabla_{v} f_{\alpha}=0,
$$

$$
\begin{array}{ll}
E_{t}=c \operatorname{curl} B-j ; & \nabla \cdot E=\rho ; \\
B_{t}=-c \operatorname{curl} E ; & \nabla \cdot B=0 .
\end{array}
$$

These are the relativistic Vlasov-Maxwell equations (RVM). Cauchy data are prescribed

$$
f_{\alpha}(0, x, v)=f_{\alpha 0}(x, v), \quad E(0, x)=E_{0}(x), \quad B(0, x)=B_{0}(x)
$$

which satisfy the necessary constraints.

In [GS1] we have given a general sufficient condition for the existence of a global $C^{1}$-solution; namely, that there exists a continuous function $\beta(t)$ such that any solution $f_{\alpha}$ (or any iterative approximation $f_{\alpha}^{(n)}$ ) vanishes for $|v|>\beta(t)$ and all $x, \alpha, t$. In this paper we verify this condition under a smallness assumption on the data, which we state precisely as

Theorem 1. For each $k>0$, there exist constants $\varepsilon_{0}>0$ and $\beta>0$ with the following property. Let $f_{\alpha 0}(x, v)(\alpha=1,2, \ldots, N)$ be non-negative $C^{1}$ functions with supports in $\{|x| \leqq k\},\{|v| \leqq k\}$. Let $E_{0}(x), B_{0}(x)$ be $C^{2}$ functions with supports in $\{|x| \leqq k\}$ which satisfy the constraints

If the data satisfy

$$
\nabla \cdot E_{0}=\rho_{0} \equiv 4 \pi \int_{\mathbb{R}^{3}} \sum_{\alpha} e_{\alpha} f_{\alpha 0} d v, \quad \nabla \cdot B_{0}=0
$$

$$
\sum_{\alpha}\left\|f_{\alpha 0}\right\|_{C^{1}}+\left\|E_{0}\right\|_{C^{2}}+\left\|B_{0}\right\|_{C^{2}} \leqq \varepsilon_{0}
$$

then there exists a unique solution of RVM for all $x \in \mathbb{R}^{3}$ and all $v \in \mathbb{R}^{3}$ and all times $t, 0 \leqq t<\infty$, with $f_{\alpha}, E, B \in C^{1}$ having initial data $f_{\alpha 0}, E_{0}, B_{0}$ such that

$$
f_{\alpha}(t, x, v)=0 \text { for }|v| \geqq \beta \text { for all } \alpha, t \text { and } x .
$$

Furthermore, $E(t, x)=B(t, x)=0$ for $|x|>c t+k$. For all $\varepsilon>0$, there exists $\varepsilon_{0}>0$ such that if (6) holds, then

$$
|E(t, x)|+|B(t, x)| \leqq \frac{\varepsilon}{(t+1)(c t-|x|+2 k)}
$$

for all $t \geqq 0, x \in \mathbb{R}^{3}$.

To our knowledge this is the first global existence theorem for RVM with assumptions only on the initial data. As mentioned above, in [GS1] we proved the global existence provided we knew a priori that the supports of $f_{\alpha}(t, x, v)$ in the momentum variable were bounded for bounded times. In [GS2] we improved this to the condition that the kinetic energy density

$$
\int\left(m_{\alpha}^{2} c^{2}+|v|^{2}\right)^{1 / 2} f_{\alpha}(t, x, v) d v
$$

be bounded for bounded times.

If the magnetic field vanishes $(B=0)$, there is an electric potential $\varphi$ satisfying $\nabla \varphi=E$ and the Maxwell equations reduce to $\Delta \varphi=\rho$. This equation coupled to the (non-relativistic) Vlasov equation is called the Vlasov-Poisson system (VP). 
Bardos and Degond [BD] solved the small data Cauchy problem for VP. The case of an external magnetic field is considered in [BDH]. Horst [H] solved a small data problem for RVM of an unusual type with data at finite and infinite times. He assumed $f_{\alpha 0}$ are small and constructed solutions of RVM which satisfy

$$
f_{\alpha}(0, x, v)=f_{\alpha 0}(x, v), \quad E(\infty, x)=0, \quad B(\infty, x)=0
$$

in the sense of scattering theory. We are indebted to Bardos, Degond and Horst for some of the key ideas used in this paper.

The key step in all these proofs is to show that the paths of the particles spread out with time. So only a small set of momenta (of diameter $O(1 / t)$ in momentum space as $t \rightarrow \infty)$ could reach a given point $(t, x)$ from the support of the initial data. Then $\rho(t, x)$, which is an integral over a set of momenta of volume $O\left(t^{-3}\right)$, is itself of that order. Since the particle paths are given by the equations

$$
\dot{x}=\hat{v}_{\alpha}, \quad \dot{v}=e_{\alpha}\left(E+c^{-1} \hat{v}_{\alpha} \wedge B\right)
$$

the particles would move in approximately straight lines if $E$ and $B$ were small. Thus we must also prove that the electromagnetic field decays as $t \rightarrow \infty$.

For the case of VP there is no explicit time dependence in the field equation $(\Delta \varphi=\rho)$ so that Bardos and Degond were able to iterate in a space where $E$ decays in $L^{\infty}$ at the rate $O\left(t^{-2}\right)$ and $\nabla E$ at the rate $O\left(t^{-5 / 2}\right)$. For RVM, the field satisfies inhomogeneous wave equations like

$$
\left(\partial_{t}^{2}-\Delta\right) E=-\nabla \rho-\partial_{t} j .
$$

So the best possible $L^{\infty}$ rate of decay for the field is $O\left(t^{-1}\right)$, which is far too slow for the methods of [BD] to succeed. Horst, on the other hand, imposed conditions as $t \rightarrow \infty$ designed to allow more rapid decay of the field. For our problem with arbitrary initial conditions and a magnetic field, we introduce a weighted $L^{\infty}$ norm for the field, as was pioneered by John $[\mathrm{J}]$. We use the weight

$$
(c t+|x|+2 k)(c t-|x|+2 k) \text {. }
$$

By causality we know that $|x| \leqq c t+k$ on the support of the field, so that the second factor simply introduces an extra decay factor of $t^{-1}$ inside the light cone. In order to use this extra factor when solving RVM, one must show that $|x|$ stays well inside the cone. Since $\dot{x}=\hat{v}_{\alpha}$ this is true if the momenta $v$ remain bounded. This means that the Vlasov and Maxwell characteristics are well separated; that is, the system RVM effectively is strictly hyperbolic. Thus we have come full circle to a condition on the behavior of the momenta. To carry out the process we also need to estimate the spatial derivatives of the field, for which another weight function is used.

We maintain the notation of [GS1]. In particular, $y \wedge z$ denotes the ordinary cross product of vectors in $\mathbb{R}^{3}$. Constants which change from line to line are simply denoted by $c$.

\section{Outline of the Proof}

The main structure of the proof follows [GS1]. Uniqueness is proved in Theorem 1 of [GS1], under condition (7). For the existence in [GS1] the following construction 
is made. Given $E^{(0)}(t, x)$ and $B^{(0)}(t, x)$, we define $E^{(n)}(t, x), B^{(n)}(t, x)$ and $f^{(n)}(t, x, v)$ inductively as follows. Given the $(n-1)^{s t}$ iteration, we define $f_{\alpha}^{(n)}$ as the solution of the linear equation,

$$
\begin{aligned}
& \partial_{t} f_{\alpha}^{(n)}+\hat{v}_{\alpha} \cdot \nabla_{x} f_{\alpha}^{(n)}+e_{\alpha}\left(E^{(n-1)}+\hat{v}_{\alpha} \wedge B^{(n-1)}\right) \cdot \nabla_{v} f_{\alpha}^{(n)}=0, \\
& \quad f_{\alpha}^{(n)}(0, x, v)=f_{\alpha 0}(x, v) .
\end{aligned}
$$

For simplicity we have set the speed of light equal to unity $(c=1)$. Then we define $\rho^{(n)}=4 \pi \sum_{\alpha} e_{\alpha} f_{\alpha}^{(n)} d v$ and $j^{(n)}=4 \pi \sum_{\alpha} e_{\alpha} \int \hat{v}_{\alpha} f_{\alpha}^{(n)} d v$. Finally we define $E^{(n)}, B^{(n)}$ as the solution of Maxwell's equations,

$$
\begin{array}{ll}
\partial_{t} E^{(n)}=\nabla \times B^{(n)}-j^{(n)}, & \nabla \cdot E^{(n)}=\rho^{(n)}, \\
\partial_{t} B^{(n)}=-\nabla \times E^{(n)}, & \nabla \cdot B^{(n)}=0,
\end{array}
$$

with data $E^{(n)}(0, x)=E_{0}(x), B^{(n)}(0, x)=B_{0}(x)$.

Theorem 1 of [GS1] states that if there exists $\beta>0$, independent of $t, x, \alpha$ and $n$, such that

$$
f_{\alpha}^{(n)}(t, x, v)=0 \quad \text { for } \quad|v|>\beta,
$$

then $\left(f_{\alpha}^{(n)}, E^{(n)}, B^{(n)}\right)$ converge to a $C^{1}$ solution $\left(f_{\alpha}, E, B\right)$ of RVM. The rest of this paper is devoted to proving $(7 n)$ under the "smallness condition" (6).

We abbreviate the field as $K(t, x)=$ the pair $(E(t, x), B(t, x))$. Define the norms

$$
\begin{aligned}
& \|K\|_{0}=\sup _{x, t}(t+|x|+2 k)(t-|x|+2 k)\{|E(t, x)|+|B(t, x)|\}, \\
& \|K\|_{1}=\sup _{x, t} \frac{(t+|x|+2 k)(t-|x|+2 k)^{2}}{\log (t+|x|+2 k)}\left\{\left|E_{x}(t, x)\right|+\left|B_{x}(t, x)\right|\right\},
\end{aligned}
$$

where $E_{x}, B_{x}$ denote the gradients of $E, B$ in the $x$ variable and $\|K\|=\|K\|_{0}+$ $\|K\|_{1}$. Let $\varepsilon>0$ and let

$$
\mathscr{K} \equiv\left\{K \mid K \quad \text { is } C^{1}, K=0 \text { for }|x|>t+k, \quad\|K\| \leqq \varepsilon\right\} .
$$

Given $K \in \mathscr{K}$, we define the characteristics as the solutions $X=X_{\alpha}(s, t, x, v)$, $V=V_{\alpha}(s, t, x, v)$ of the ordinary differential system (9), that is,

$$
\begin{aligned}
& \frac{\partial X}{\partial s}=\hat{V} \equiv V\left(m_{\alpha}^{2}+|V|^{2}\right)^{-1 / 2}, \\
& \frac{\partial V}{\partial s}=e_{\alpha}(E(s, X)+\hat{V} \wedge B(s, X)),
\end{aligned}
$$

with the "initial" conditions $X_{\alpha}(t, t, x, v)=x$ and $V_{\alpha}(t, t, x, v)=v$.

Next we define

$$
f_{\alpha}(t, x, v)=f_{\alpha 0}\left(X_{\alpha}(0, t, x, v), \quad V_{\alpha}(0, t, x, v)\right) .
$$

Thus $f_{\alpha}(t, x, v)$ is the solution of the Vlasov equation,

$$
\partial_{t} f_{\alpha}+\hat{v}_{\alpha} \cdot \nabla_{x} f_{\alpha}+e_{\alpha}\left(E+\hat{v}_{\alpha} \wedge B\right) \cdot \nabla_{v} f_{\alpha}=0
$$

with the initial condition $f_{\alpha}(0, x, v)=f_{\alpha 0}(x, v)$. We define $\rho$ and $j$ by (1) and (2) and 
define $K^{*}=\left(E^{*}, B^{*}\right)$ as the solution of Maxwell's equations,

$$
\begin{array}{ll}
\partial_{t} E^{*}=\nabla \times B^{*}-j ; & \nabla \cdot E^{*}=\rho, \\
\partial_{t} B^{*}=-\nabla \times E^{*} ; & \nabla \cdot B^{*}=0,
\end{array}
$$

with the initial conditions $E^{*}(0, x)=E_{0}(x), B^{*}(0, x)=B_{0}(x)$.

Thus the iteration scheme may be summarized as $K^{(n)}=\left(K^{(n-1)}\right)^{*}$. We begin the scheme by defining $K^{(0)} \equiv 0$ (that is, $E^{(0)}(t, x) \equiv B^{(0)}(t, x) \equiv 0$ ). We shall prove the following two theorems.

Theorem 2. If $K \in \mathscr{K}$ and $\varepsilon$ is small enough, then there exists $\beta>0$ depending only on $k, \varepsilon$ and $\varepsilon_{0}$ such that $f_{\alpha}(t, x, v)=0$ for $|v| \geqq \beta$ and for all $\alpha, x, t$.

Theorem 3. If $K \in \mathscr{K}$ and $\varepsilon$ is small enough, then $K^{*} \in \mathscr{K}$.

Proof of Theorem 1. We define the sequences $f_{\alpha}^{(n)}, K^{(n)}$ as above. Since $K^{(0)} \in \mathscr{K}$, Theorem 3 states that $K^{(n)} \in \mathscr{K}$ for all $n$. By Theorem $2, f_{\alpha}^{(n)}=0$ for $|v| \geqq \beta$. By [GS1], $f_{\alpha}^{(n)}, K^{(n)}$ and their first derivatives converge pointwise to $f$ and $K$. Therefore $K \in \mathscr{K}$. Hence (8) is valid, as well as

$$
\left|\nabla_{x} E(t, x)\right|+\left|\nabla_{x} B(t, x)\right| \leqq \frac{\varepsilon \log (2+t)}{(1+t)(t-|x|+2 k)^{2}}
$$

for all $t \geqq 0, x \in \mathbb{R}^{3}$. As we mentioned earlier, $(f, K)$ is a solution of RVM.

\section{The Characteristics}

The characteristics are the curves defined by (15) and (16). They exist as $C^{1}$ functions of $s, t, x, v$ for some time $0 \leqq t<T^{*}, 0 \leqq s<T^{*}$ because $E$ and $B$ are $C^{1}$ functions. For as long as the characteristics exist, we define

$$
P(t)=\sup \left\{\left|V_{\alpha}(s, 0, x, v)\right|:|x| \leqq k,|v| \leqq k, 0 \leqq s \leqq t, 1 \leqq \alpha \leqq N\right\},
$$

that is, the largest momentum up to time $t$ emanating from the support of $f_{\alpha 0}$. Then $P(t)$ is a continuous function of $t$ for $0 \leqq t<T^{*}$.

Lemma 1. If $0 \leqq s \leqq t$ and $f_{\alpha}(t, x, v) \neq 0$, then

$$
s-\left|X_{\alpha}(s, t, x, v)\right|+2 k \geqq(k+s)\left(2+2 P^{2}(t)\right)^{-1} .
$$

Proof. If $0 \leqq s \leqq t,\left|X_{\alpha}(0, t, x, v)\right| \leqq k$ and $\left|V_{\alpha}(0, t, x, v)\right| \leqq k$, we have

Hence $|v| \leqq P(t)$ and

$$
\left|V_{\alpha}(s, t, x, v)\right| \leqq P(t) .
$$

$$
\left|X_{\alpha}(s, t, x, v)\right| \leqq\left|X_{\alpha}(0, t, x, v)\right|+\int_{0}^{s}\left|\hat{V}_{\alpha}(\tau, t, x, v)\right| d \tau \leqq k+s \hat{P}(t),
$$

where, assuming $m_{\alpha}=e_{\alpha}=1$ for simplicity,

But

$$
\hat{P}(t)=P(t)\left(1+P^{2}(t)\right)^{-1 / 2}<1 .
$$

$$
1-\hat{P}(t)=\left(1+P^{2}(t)\right)^{-1 / 2}\left\{\left(1+P^{2}(t)\right)^{1 / 2}+P\right\}^{-1} \geqq \frac{1}{2\left(1+P^{2}(t)\right)} .
$$


So

$$
\left|X_{\alpha}(s, t, x, v)\right| \leqq k+s-s\left\{2\left(1+P^{2}(t)\right)\right\}^{-1}
$$

which implies Lemma 1.

Lemma 2. If $K \in \mathscr{K}$ and if $\varepsilon$ is sufficiently small, say $\varepsilon<\varepsilon_{1}$, then the characteristics $X_{\alpha}(s), V_{\alpha}(s)$ exist for all $s\left(T^{*}\right.$ is infinite) and $P(t)$ is bounded, say $P(t) \leqq \beta$. Here $\varepsilon_{1}$ and $\beta$ depend only on $k$. Therefore, if $f_{\alpha}(t, x, v) \neq 0$ for some $(\alpha, t, x)$, then $|v| \leqq \beta$.

Proof. We have for $t \geqq 0$, writing $X(s)=X_{\alpha}(s, t, x, v)$ and $V(s)=V_{\alpha}(s, t, x, v)$,

$$
\begin{aligned}
\left|V_{\alpha}(0, t, x, v)-v\right| & \leqq \int_{0}^{t}|E(s, X(s))+\hat{V}(s) \wedge B(s, X(s))| d s \\
& \leqq\|K\|_{0} \int_{0}^{t}(s+|X(s)|+2 k)^{-1}(s-|X(s)|+2 k)^{-1} d s \\
& \leqq\|K\|_{0} \int_{0}^{t}(s+k)^{-2}\left(2+2 P^{2}(t)\right) d s \\
& \leqq 2 k^{-1}\left(1+P^{2}(t)\right)\|K\|_{0},
\end{aligned}
$$

by Lemma 1 , provided $\left|X_{\alpha}(0, t, x, v)\right| \leqq k$ and $\left|V_{\alpha}(0, t, x, v)\right| \leqq k$. Let $y=X_{\alpha}(0, t, x, v)$ and $w=V_{\alpha}(0, t, x, v)$, so that $x=X_{\alpha}(t, 0, y, w)$ and $v=V_{\alpha}(t, 0, y, w)$. Then

$$
\left|w-V_{\alpha}(t, 0, y, w)\right| \leqq 2 k^{-1}\left(1+P^{2}(t)\right)\|K\|_{0}
$$

for $|w| \leqq k$ and $|y| \leqq k$. Thus

$$
P(t) \leqq k+2 k^{-1}\left(1+P^{2}(t)\right)\|K\|_{0} .
$$

If $\|K\|_{0}$ is sufficiently small (depending on $k$ ), this implies that $P(t)$ is a bounded function of $t$.

Proof of Theorem 2. By Lemma 2, the characteristics exist for all time. If $f_{\alpha}(t, x, v) \neq 0$ for some $(x, t, \alpha, v)$, then by (17) $|y|=\left|X_{\alpha}(0, t, x, v)\right| \leqq k$ and $|w|=$ $\left|V_{\alpha}(0, t, x, v)\right| \leqq k$. By the definition of $P(t)$,

$$
|v|=\left|V_{\alpha}(t, 0, y, w)\right| \leqq P(t) \leqq \beta .
$$

Lemma 3. If $\varepsilon$ is sufficiently small, there is a constant $c>0$ so that

$$
\left|X_{\alpha}(0, t, x, v)-X_{\alpha}(0, t, x, w)\right| \geqq c t|v-w|
$$

for all $t, x, v$ and $w$ such that $f_{\alpha}(t, x, v) \neq 0$ and $f_{\alpha}(t, x, w) \neq 0$.

Proof. For simplicity, since $\alpha$ is fixed, we drop the subscript $\alpha$. Following Horst $[\mathrm{H}]$, we rewrite the characteristic equation for $d v / d t$ in terms of $\hat{v}$, as follows:

$$
\frac{d \hat{v}}{d t}=m^{-1}\left(1-|\hat{v}|^{2}\right)^{1 / 2}\{E+\hat{v} \wedge B-(\hat{v} \cdot E) \hat{v}\} \equiv J(t, x, \hat{v})
$$

Since $\left(1-|\hat{v}|^{2}\right)^{1 / 2}=m\left(m^{2}+|v|^{2}\right)^{-1 / 2}<1$, the derivatives of $J$ can be estimated as

$$
\begin{aligned}
\left|\nabla_{x} J(t, x, \hat{v})\right| & \leqq c\left(1-|\hat{v}|^{2}\right)^{1 / 2}\left|\nabla_{x} K\right| \\
& \leqq c\|K\|_{1}(t+r+2 k)^{-1} \log (t+r+2 k)(t-r+2 k)^{-2},
\end{aligned}
$$


where $r=|x|$, and

$$
\begin{aligned}
\left|\nabla_{\hat{v}} J(t, x, \hat{v})\right| & \leqq c\left(1-|\hat{v}|^{2}\right)^{-1 / 2}|K| \leqq c\left(1+|v|^{2}\right)^{1 / 2}|K| \\
& \leqq c\left(1+|v|^{2}\right)^{1 / 2}\|K\|_{0}(t+r+2 k)^{-1}(t-r+2 k)^{-1}
\end{aligned}
$$

For the characteristic passing through the point $(t, x, v)$, where $f(t, x, v) \neq 0$, we abbreviate $X(s)=X(s, t, x, v)$ and $V(s)=V(s, t, x, v)$. Then we substitute $s$ for $t, X(s)$ for $x$ and $V(s)$ for $v$ to obtain

$$
\left|\left(\nabla_{\hat{v}} J\right)(s, X(s), \hat{V}(s))\right| \leqq c\left(1+\beta^{2}\right)^{2}\|K\|(k+s)^{-3} \log (k+s) \equiv g(s),
$$

and

$$
\left|\left(\nabla_{\hat{v}} J\right)(s, X(s), \hat{V}(s))\right| \leqq c\left(1+\beta^{2}\right)^{3 / 2}\|K\|(k+s)^{-2} \equiv h(s)
$$

\section{by Lemma 1 .}

Now let $t, x, v$ and $w$ be fixed so that $f(t, x, v) \neq 0$ and $f(t, x, w) \neq 0$. For brevity we denote $X_{1}(s)=X(s, t, x, v)$ and $X_{2}(s)=X(s, t, x, w)$. By (15) and (21) we have, for $0 \leqq \xi \leqq t$

$$
\begin{aligned}
X_{1}(\xi)-X_{2}(\xi)+(t-\xi)(\hat{v}-\hat{w}) & =\int_{\xi}^{t}\left[-\hat{V}_{1}(\tau)+\hat{v}+\hat{V}_{2}(\tau)-\hat{w}\right] d \tau \\
& =\int_{\xi}^{t} \int_{\tau}^{t}\left[\frac{\partial \hat{V}_{1}}{\partial s}-\frac{\partial \hat{V}_{2}}{\partial s}\right] d s d \tau \\
& =\int_{\xi}^{t} \int_{\tau}^{t}\left[J\left(s, X_{1}(s), \hat{V}_{1}(s)\right)-J\left(s, X_{2}(s), \hat{V}_{2}(s)\right)\right] d s d \tau
\end{aligned}
$$

Hence by (22) and (23), we have

$$
\left|X_{1}(\xi)-X_{2}(\xi)+(t-\xi)(\hat{v}-\hat{w})\right| \leqq D(\xi),
$$

where

$$
\begin{aligned}
D(\xi) \equiv & \int_{\xi}^{t}\left|-\hat{V}_{1}(\tau)+\hat{v}+\hat{V}_{2}(\tau)-\hat{w}\right| d \tau \\
\leqq & \int_{\xi}^{t} \int_{\tau}^{t}\left\{g(s)\left|X_{1}(s)-X_{2}(s)\right|+h(s)\left|\hat{V}_{1}(s)-\hat{V}_{2}(s)\right|\right\} d s d \tau \\
\leqq & \int_{\xi}^{t} \int_{\tau}^{t}\left\{g(s) \int_{s}^{t}\left|\hat{V}_{1}(\theta)-\hat{V}_{2}(\theta)\right| d \theta+h(s)\left|\hat{V}_{1}(s)-\hat{V}_{2}(s)\right|\right\} d s d \tau \\
\leqq & \int_{\xi}^{t}\left[\int_{\tau}^{t} g(s)\{D(s)+(t-s)|\hat{v}-\hat{w}|\} d s+h(\tau) D(\tau)\right. \\
& \left.\quad+\int_{\tau}^{t} h(s) d s|\hat{v}-\hat{w}|\right] d \tau .
\end{aligned}
$$

In the first part of this expression, we switch the order of integration, obtaining the factor $(s-\xi) \leqq s$. In terms of

$$
I=\int_{0}^{\infty}[s g(s)+h(s)] d s,
$$


we therefore find the estimate

$$
D(\xi) \leqq \int_{\xi}^{t}[s g(s)+h(s)] D(s) d s+(t-\xi)|\hat{v}-\hat{w}| I .
$$

After changing variables $\xi \rightarrow(t-\xi)$, we apply Gronwall's inequality to obtain

$$
D(\xi) \leqq(t-\xi)|\hat{v}-\hat{w}| I \exp \int_{\xi}^{t}[s g(s)+h(s)] d s \leqq(t-\xi)|\hat{v}-\hat{w}| I \exp I .
$$

We choose $\varepsilon$ so small that $I \exp I<1 / 2$, recalling that $\|K\|<\varepsilon$. Choosing $\xi=0$, we therefore have

$$
\left|X_{1}(0)-X_{2}(0)+t(\hat{v}-\hat{w})\right| \leqq D(0) \leqq(t / 2)|\hat{v}-\hat{w}| .
$$

Therefore

$$
\left|X_{1}(0)-X_{2}(0)\right| \geqq(t / 2)|\hat{v}-\hat{w}| .
$$

Now $v=m \hat{v}\left(1-|\hat{v}|^{2}\right)^{-1 / 2}$, so that

$$
\left|\partial v_{i} / \partial \hat{v}_{j}\right| \leqq c\left(1-|\hat{v}|^{2}\right)^{-3 / 2}=c\left(m^{2}+|v|^{2}\right)^{+3 / 2}
$$

for each $i$ and $j$. Since $f(t, x, v) \neq 0$, we have $\left|X_{1}(0)\right| \leqq k$ and $\left|V_{1}(0)\right| \leqq k$, whence $|v| \leqq P(t) \leqq \beta$ by (20) and Lemma 2 . Similarly $|w| \leqq \beta$. Therefore

$$
|v-w| \leqq c(1+\beta)^{3}|\hat{v}-\hat{w}| \text {. }
$$

By (24), we have $\left|X_{1}(0)-X_{2}(0)\right| \geqq c t|v-w|$.

\section{The Particle Densities}

We assume that $\varepsilon$ is small enough for the validity of Lemmas 2 and 3 .

Lemma 4. There is a positive constant $c$ such that

$$
\int f_{\alpha}(t, x, v) d v \leqq c\left\|f_{0}\right\|_{0}(1+t)^{-3} \leqq c\left\|f_{0}\right\|_{0}(t+|x|+2 k)^{-3}
$$

for all $t \geqq 0$, where $\left\|f_{0}\right\|_{0} \equiv \sup _{\alpha, x, v}\left|f_{\alpha 0}(x, v)\right|$.

Proof. We recall that $f_{\alpha}(t, x, v)=f_{\alpha 0}(X, V)$, where $X=X_{\alpha}(0, t, x, v)$ and $V=V_{\alpha}$ $(0, t, x, v)$. We have $|X| \leqq k$ and $|V| \leqq k$ so'that $|v| \leqq \beta$ by Lemma 2 . So Lemma 4 is valid for $t \leqq 1$. Now the integration in $\int f_{\alpha} d v$ may be taken over the set $\mathscr{A}=\{v:|X| \leqq k\}$. By Lemma 3 the diameter of $\mathscr{A}$ is at most $2 k /(c t)$, and so its volume is at most $O\left(t^{-3}\right)$. Therefore $\int f_{\alpha} d v$ is at most $c\left\|f_{0}\right\|_{0} t^{-3}$. The last inequality follows from the vanishing of $f_{\alpha}(t, x, v)$ for $|x|>t+k$.

Lemma 5. There is a positive constant $c$ such that

and

$$
\left|\nabla_{x} f_{\alpha}(t, x, v)\right| \leqq c\left\|f_{0}\right\|_{1}
$$

$$
\left|\nabla_{v} f_{\alpha}(t, x, v)\right| \leqq c(1+t)\left\|f_{0}\right\|_{1},
$$

where $\left\|f_{0}\right\|_{1}=\sup _{\alpha, x, v}\left\{\left|f_{\alpha 0}(x, v)\right|+\left|\nabla_{x} f_{\alpha 0}(x, v)\right|+\left|\nabla_{v} f_{\alpha 0}(x, v)\right|\right\}$. 
Proof. Write $L_{\alpha}=\partial_{t}+\hat{v}_{\alpha} \cdot \nabla_{x}+\left(E+\hat{v}_{\alpha} \wedge B\right) \cdot \nabla_{v}$, so that the Vlasov equation is $L_{\alpha} f_{\alpha}=0$. Again we drop the subscript $\alpha$. Write $X(s)=X(s, t, x, v)$ and $V(s)=$ $V(s, t, x, v)$ as before. Fix a coordinate $x_{j}$ and let $\partial f=\partial f / \partial x_{j}$ for brevity. Then

Thus

$$
L(\partial f)=-(\partial E+\hat{v} \wedge \partial B) \cdot \nabla_{v} f \equiv h(t, x, v)
$$

$$
\frac{d}{d s}[\partial f(s, X(s), V(s))]=h(s, X(s), V(s)) .
$$

Integrating from 0 to $\tau \leqq t$, we have

so that

$$
\partial f(\tau, X(\tau), V(\tau))=\partial f_{0}(X(0), V(0))+\int_{0}^{\tau} h(s, X(s), V(s)) d s
$$

$$
\begin{aligned}
\left|\nabla_{x} f(\tau, X(\tau), V(\tau))\right| & \leqq\left\|f_{0}\right\|_{1}+\|K\|_{1} \int_{0}^{\tau} \frac{\log (s+|X(s)|+2 k)\left|\nabla_{v} f(s, X(s), V(s))\right|}{(s+|X(s)|+2 k)(s-|X(s)|+2 k)^{2}} d s \\
& \leqq\left\|f_{0}\right\|_{1}+c \int_{0}^{\tau} \frac{\log (2+s)}{(1+s)^{3}}\left|\nabla_{v} f(s, X(s), V(s))\right| d s
\end{aligned}
$$

by Lemmas 1 and 2 . We write this for short as

$$
\left|\nabla_{x} f(\tau)\right| \leqq\left\|f_{0}\right\|_{1}+c \int_{0}^{\tau} I(s)\left|\nabla_{v} f(s)\right| d s .
$$

Now let $D f=\partial f / \partial v_{j}$ for brevity. Then

$$
|L(D f)|=\left|-(D \hat{v}) \cdot\left(\nabla_{x} f+B \wedge \nabla_{v} f\right)\right| \leqq\left|\nabla_{x} f\right|+|B|\left|\nabla_{v} f\right| .
$$

Integrating as above, we have

$$
\begin{aligned}
\left|\nabla_{v} f(\tau, X(\tau), V(\tau))\right| \leqq & \left\|f_{0}\right\|_{1}+\int_{0}^{\tau}\left\{\left|\nabla_{x} f\right|+|B|\left|\nabla_{v} f\right|\right\}(s, X(s), V(s)) d s \\
\leqq & \left\|f_{0}\right\|_{1}+\int_{0}^{\tau}\left|\nabla_{x} f\right| d s \\
& +\|K\|_{0} \int_{0}^{\tau} \frac{\left|\nabla_{v} f\right| d s}{(s+|X(s)|+2 k)(s-|X(s)|+2 k)},
\end{aligned}
$$

where $\nabla_{x} f$ and $\nabla_{v} f$ are evaluated at $(s, X(s), V(s))$. The last kernel is at most $c(1+s)^{-2}$, as above. Since it is integrable, Gronwall's inequality yields

$$
\left|\nabla_{v} f(\tau)\right| \leqq c\left[\left\|f_{0}\right\|_{1}+\int_{0}^{\tau}\left|\nabla_{x} f(s)\right| d s\right] .
$$

We now have (25) and (26), where $I(s)=(1+s)^{-3} \log (2+s)$. Substituting (25) into (26) we have

$$
\left|\nabla_{v} f(\tau)\right| \leqq c c_{0}(1+\tau)+c^{2} \int_{0}^{\tau}(\tau-s) I(s)\left|\nabla_{v} f(s)\right| d s,
$$

where $c_{0}=\left\|f_{0}\right\|_{1}$. Therefore

$$
g(\tau) \equiv(1+\tau)^{-1}\left|\nabla_{v} f(\tau)\right| \leqq c_{0} c_{1}+c^{2} \int_{0}^{\tau}(1+s) I(s) g(s) d s
$$


Since $(1+s) I(s)$ is also integrable, we deduce by Gronwall again that $g(\tau)$ is bounded. By (25), $\left|\nabla_{x} f\right|$ is also bounded for $0 \leqq \tau \leqq t$. The bounds are independent of $\tau, t, x$ and $v$. Putting $\tau=t$ we conclude that

and

$$
\left|\nabla_{x} f(t, x, v)\right|=\left|\nabla_{x} f(t, X(t), V(t))\right| \leqq c
$$

$$
\left|\nabla_{v} f(t, x, v)\right|=\left|\nabla_{v} f(t, X(t), V(t))\right| \leqq c(1+t)
$$

\section{The Field}

As we showed above, the characteristics, defined by (15) and (16), exist globally. Because $|\partial X / \partial s|=|\widehat{V}|<1$, and $f_{\alpha 0}$ has support in $\{|x| \leqq k\}$, the particle density $f_{\alpha}(t, x, v)$ defined by (17) has support in $|x| \leqq t+k$. Therefore so do $j$ and $\rho$. Therefore $E^{*}$ and $B^{*}$, defined by (19), also have supports in $|x| \leqq t+k$. In order to prove Theorem 3 , it remains to show that $\left\|K^{*}\right\|<\varepsilon$ for $\varepsilon$ and $\varepsilon_{0}$ sufficiently small.

Lemma 6. There is a constant $c>0$ so that

$$
\left\|K^{*}\right\|_{0} \leqq c \varepsilon_{0}\left(1+\|K\|_{0}\right)
$$

for all $K \in \mathscr{K}$ provided $\varepsilon$ is sufficiently small.

Proof. We begin with the representation formula for $E^{*}$ from Theorem 3 of [GS1]; namely,

$$
E^{*}=E_{z}^{*}+E_{T}^{*}+E_{S}^{*}
$$

where the terms are given explicitly as follows:

$$
\begin{aligned}
E_{S}^{*}(t, x) & =-\sum_{\alpha} e_{\alpha} \int_{|y-x| \leqq t} \int \frac{\omega+\hat{v}_{\alpha}}{1+\hat{v}_{\alpha} \cdot \omega} S_{\alpha} f_{\alpha} d v \frac{d y}{|y-x|} \\
& =-\sum_{\alpha} e_{\alpha}^{2} \int_{|y-x| \leqq t} \int \nabla_{v}\left[\frac{\omega+\hat{v}_{\alpha}}{1+\hat{v}_{\alpha} \cdot \omega}\right] \cdot\left(E+\hat{v}_{\alpha} \wedge B\right) f_{\alpha} d v \frac{d y}{|y-x|},
\end{aligned}
$$

where $f_{\alpha}=f_{\alpha}(t-|y-x|, y, v), \quad E=E(t-|y-x|, y), \quad B=B(t-|y-x|, y), \quad \omega=$ $(y-x) /|y-x|$ and $S_{\alpha}=\partial_{t}+\hat{v}_{x} \cdot \nabla_{x}$ by Eq. (55) in [GS1].

$$
E_{T}^{*}(t, x)=-\sum_{\alpha} e_{\alpha}^{\cdot} \int_{|y-x| \leqq t} \int_{0} \frac{\left(\omega+\hat{v}_{\alpha}\right)\left(1-\left|\hat{v}_{\alpha}\right|^{2}\right)}{\left(1+\hat{v}_{\alpha} \cdot \omega\right)^{2}} f_{\alpha} d v \frac{d y}{|y-x|^{2}} .
$$

The term $E_{z}^{*}$ is the sum of the solution $\mathscr{E}(t, x)$ of the homogeneous ("free") Maxwell equations with the same initial data and of the boundary term from Eqs. (21)-(22) of [GS1]. The latter involves the expression

Therefore

$$
\sum_{j} \omega_{j} a_{j}=\frac{\omega_{i}-\left(\hat{v}_{\alpha} \cdot \omega\right) \hat{v}_{\alpha i}}{1+\hat{v}_{\alpha} \cdot \omega}
$$

where

$$
E_{z}^{*}(t, x)=\mathscr{E}(t, x)-\sum_{\alpha} \frac{e_{\alpha}}{t} \int_{|y-x|=t} \int \frac{\omega-\left(\hat{v}_{\alpha} \cdot \omega\right) \hat{v}_{\alpha}}{1+\hat{v}_{\alpha} \cdot \omega} f_{\alpha 0}(y, v) d v d S_{y},
$$

$$
\mathscr{E}(t, x)=\frac{1}{4 \pi t^{2}} \int_{|y-\lambda|=t}\left[E_{0}(y)+((y-x) \cdot \nabla) E_{0}(y)+t \nabla \times B_{0}(y)\right] d S_{y} .
$$


Now $E_{z}^{*}$ is easy to estimate. Both of its terms have supports in $|t-| x|| \leqq k$ because $f_{\alpha 0}(y, v), E_{0}(y)$ and $B_{0}(y)$ have supports in $|y| \leqq k$. Furthermore, both of them have a factor $t^{-1}$. Therefore we can replace $t^{-1}$ by $(t+|x|+2 k)^{-1}$, and we can insert arbitrary factors of $t-|x|+2 k$. Hence

$$
\left\|E_{z}^{*}\right\|_{0} \leqq c\left(\left\|E_{0}\right\|_{C^{1}}+\left\|B_{0}\right\|_{C^{1}}+\left\|f_{0}\right\|_{C^{0}}\right) \text {. }
$$

As for the main terms $E_{T}^{*}$ and $E_{S}^{*}$, we note that the kernels are bounded because $|v| \leqq \beta$ (by Theorem 2) and $|\omega|=1$. Thus

$$
\begin{aligned}
\left|E_{T}^{*}(t, x)\right| & \leqq c \sum_{\alpha} \int_{|y-\chi| \leqq t} \int f_{\alpha}(t-|x-y|, y, v) d v \frac{d y}{|y-x|^{2}} \\
& \leqq c \varepsilon_{0} \int_{|y-x| \leqq t}(t-|x-y|+|y|+2 k)^{-3}|y-x|^{-2} \varphi d y
\end{aligned}
$$

where $\varphi=1$ for $|y|<t-|x-y|+k$ and $\varphi=0$ for $|y|>t-|x-y|+k$, since $f_{\alpha}(\tau, y, v)=0$ for $|y|>\tau+k$ and $\int f_{\alpha}(\tau, x, v) d v \leqq c\left\|f_{0}\right\|_{0}(1+\tau)^{-3} \leqq c \varepsilon_{0}(\tau+|y|+2 k)^{-3}$ by Lemma 4 . Similarly

$$
\begin{aligned}
\left|E_{S}^{*}(t, x)\right| \leqq & c \sum_{\alpha} \int_{|y-x| \leqq t} \int f_{\alpha}(t-|x-y|, y, v) d v|K(t-|x-y|, y)| \frac{d y}{|y-x|} \\
\leqq & c \varepsilon_{0}\|K\|_{0} \int_{|y-x| \leqq t}(t-|x-y|+|y|+2 k)^{-4} \\
& \cdot(t-|x-y|-|y|+2 k)^{-1}|y-x|^{-1} \varphi d y .
\end{aligned}
$$

By Lemma 7 below, we can reduce these triple integrals to double integrals:

$$
\begin{gathered}
\left|E_{T}^{*}(t, x)\right| \leqq \frac{c \varepsilon_{0}}{r} \int_{0}^{t} \int_{a}^{b}(\tau+\lambda+2 k)^{-3} \lambda d \lambda \frac{d \tau}{t-\tau}, \\
\left|E_{S}^{*}(t, x)\right| \leqq \frac{c \varepsilon_{0}\|K\|_{0}}{r} \int_{0}^{t} \int_{a}^{b}(\tau+\lambda+2 k)^{-4}(\tau-\lambda+2 k)^{-1} \lambda \varphi d \lambda d \tau,
\end{gathered}
$$

where $\varphi=0$ for $\lambda>\tau+k, a=|r-t+\tau|, b=r+t-\tau, r=|x|, \lambda=|y|$ and $\tau=t-$ $|x-y|$. It suffices to show that $\left|E_{T}^{*}(t, x)\right| \leqq c \varepsilon_{0}(t+r+2 k)^{-1}(t-r+2 k)^{-1}$ and $\left|E_{S}^{*}(t, x)\right| \leqq c \varepsilon_{0}\|K\|_{0}(t+r+2 k)^{-1}(t-r+2 k)^{-1}$, plus the same estimates for $B^{*}(t, x)$, which are very similar.

We continue our analysis with the integral in (31). We estimate $(\tau+\lambda+2 k)^{-1} \lambda \leqq 1$ and $(\tau-\lambda+2 k)^{-1} \varphi \leqq k^{-1}$. Then the integral in (31) is less than

$$
\begin{aligned}
\frac{1}{k} \int_{0}^{t} \int_{a}^{b}(\tau+\lambda+2 k)^{-3} d \lambda d \tau= & \frac{1}{k} \int_{0}^{t} \frac{(b-a)(b+a+2 \tau+4 k)}{(\tau+a+2 k)^{2}(\tau+b+2 k)^{2}} d \tau \\
= & \frac{4 r}{k} \int_{0}^{(t-r)_{+}} \frac{t+2 k}{(t-r+2 k)^{2}(t+r+2 k)^{2}} d \tau \\
& +\frac{2}{k} \int_{(t-r)_{+}}^{t} \frac{(t-\tau)(2 r+2 \tau+4 k) d \tau}{(2 \tau+r-t+2 k)^{2}(t+r+2 k)^{2}} .
\end{aligned}
$$

The first integral is at most $c r(t-r+2 k)^{-1}(t+r+2 k)^{-1}$. In the second one, we 
use $t-\tau \leqq r$ and $2 r+2 \tau+4 k \leqq 2(t+r+2 k)$, so we get at most

$$
\frac{c r}{t+r+2 k} \int_{(t-r)_{+}}^{t} \frac{d \tau}{(2 \tau+r-t+2 k)^{2}} \leqq \frac{c r}{t+r+2 k} \int_{(t-r)_{+}}^{\infty} \frac{d \tau}{(\tau+2 k)^{2}},
$$

which is also less than $c r(t-r+2 k)^{-1}(t+r+2 k)^{-1}$, since $r \leqq t+k$.

The integral in (30) is also broken up at $\tau=(t-r)_{+}$. For $\tau \leqq(t-r)_{+}$, we write $\lambda \leqq b=r+t-\tau \leqq 2(t-\tau)$ in order to reduce it to one of the integrals already estimated. On the other hand, for $\tau \geqq(t-r)_{+}$, we estimate $\lambda \leqq \tau+\lambda+2 k$ to obtain

$$
\int_{(t-r)_{+}}^{t} \int_{a}^{b} \frac{d \lambda d \tau}{(\tau+2 k)^{2}(t-\tau)} \leqq 2 \int_{(t-r)_{+}}^{t} \frac{d \tau}{(\tau+2 k)^{2}} \leqq 2 r(t-r+2 k)^{-1}(t+2 k)^{-1},
$$

since $r \leqq t+k$. Thus both integrals in (30) and (31) are $O\left(r(t+r+2 k)^{-1}(t-\right.$ $r+2 k)^{-1}$ ). This establishes the required estimates for $E^{*}$ and essentially completes the proof of Lemma 6 since the estimates for $B^{*}$ are identical.

Indeed, from Theorem 3 of [GS1], we have (for a single species, say) the representation

$$
B^{*}=B_{z}^{*}+B_{T}^{*}+B_{S}^{*},
$$

where $B_{z}^{*}$ is given by a formula similar to (28) (and hence depends only on the data), and

$$
\begin{aligned}
B_{T}^{*} & =\iint_{|y-x| \leqq t} \frac{(\omega \wedge \hat{v})\left(1-|\hat{v}|^{2}\right)}{(1+\hat{v} \cdot \omega)^{2}} f(t-|y-x|, y, v) d v \frac{d y}{|y-x|^{2}} ; \\
\left(B_{S}^{*}\right)^{i} & =\iint_{|y-x| \leqq t} \frac{(\omega \wedge \hat{v})_{i}}{1+\hat{v} \cdot \omega}(S f)(t-|y-x|, y, v) d v \frac{d y}{|y-x|} \\
& =\iint_{|y-x| \leqq t} \nabla_{v}\left[\frac{(\omega \wedge \hat{v})_{i}}{1+\hat{v} \cdot \omega}\right] \cdot(E+\hat{v} \wedge B) f d v \frac{d y}{|y-x|} .
\end{aligned}
$$

Again the kernels appearing here are bounded because $|v| \leqq \beta$ by Theorem 2 and $|\omega|=1$. Hence $B_{T}^{*}$ and $B_{S}^{*}$ too are estimable exactly as in (30) and (31).

Lemma 7. For any continuous function $g(\tau, \lambda)$ of two real variables, and $h(\sigma)$ of one real variable,

$$
\int_{|y-x| \leqq t} g(t-|x-y|,|y|) h(|x-y|) d y=\frac{2 \pi}{|x|} \int_{0}^{t} \int_{|| x|-t+\tau|}^{|x|+t-\tau} g(\tau, \lambda) \lambda d \lambda(t-\tau) h(t-\tau) d \tau,
$$

where the integration on the left is over a ball in $\mathbb{R}^{3}$ and $c$ is an absolute constant.

Proof. The left side can be written as

$$
\int_{0|x-y|=t-\tau}^{t} \int_{|c|} g(\tau,|y|) d S_{y} h(t-\tau) d \tau
$$

So it suffices to show

$$
\int_{|x-y|=t-\tau} g(\tau,|y|) d S_{y}=\frac{2 \pi(t-\tau)}{|x|} \int_{|x|-t+\tau \mid}^{|x|+t-\tau} g(\tau, \lambda) \lambda d \lambda
$$


That is, for any function $f$,

$$
\int_{|x-y|=\sigma} f(|y|) d S_{y}=\frac{2 \pi \sigma}{|x|} \int_{|| x|-\sigma|}^{|x|+\sigma} \lambda f(\lambda) d \lambda .
$$

By symmetry we may take $x=(0,0, r)$. Then

$$
\begin{aligned}
\int_{|y-x|=\sigma} f(|y|) d S_{y} & =\sigma^{2} \int_{|\omega|=1} f(|x+\sigma \omega|) d \omega \\
& =2 \pi \sigma^{2} \int_{0}^{\pi} \sin \varphi f\left(\left(r^{2}+\sigma^{2}+2 r \sigma \cos \varphi\right)^{1 / 2}\right) d \varphi .
\end{aligned}
$$

Changing variables now by $\lambda=\left(r^{2}+\sigma^{2}+2 r \sigma \cos \varphi\right)^{1 / 2}$, we obtain the desired result.

\section{Derivatives of the Field}

In this section we shall estimate $\left\|K^{*}\right\|_{1}$. Lemma 8 (below) together with Lemma 6 will provide the bound

$$
\left\|K^{*}\right\| \leqq c \varepsilon_{0}\left(1+\|K\|+\|K\|^{2}\right)
$$

Therefore if $\|K\| \leqq \varepsilon$, if $\varepsilon$ is sufficiently small for the validity of the previous estimates, if $\varepsilon<1 / 2$ and if $\varepsilon_{0}<\varepsilon /(2 c)$, then $\left\|K^{*}\right\|<\varepsilon$. As remarked earlier, this will prove Theorem 3 and therefore Theorem 1.

Lemma 8. There is a constant $c$ such that

$$
\left\|K^{*}\right\|_{1} \leqq c \varepsilon_{0}\left(1+\|K\|_{1}^{2}\right) .
$$

Proof. We differentiate the representation formula (27) for $E^{*}$, repeating the technique of [GS1]. Thus the derivative $\partial / \partial x_{k}$ of the $i$ th component of $E^{*}$ is

$$
\begin{aligned}
\partial_{k} E^{* i}(t, x)= & \partial_{k} E_{z}^{* i}-\sum_{\alpha} e_{\alpha} \iint \frac{\left(\omega_{i}+\hat{v}_{\alpha i}\right)\left(1-\left|\hat{v}_{\alpha}\right|^{2}\right)}{\left(1+\hat{v}_{\alpha} \cdot \omega\right)^{2}} \partial_{k} f_{\alpha} d v \frac{d y}{|y-x|^{2}} \\
& -\sum_{\alpha} e_{\alpha}^{2} \iint \nabla_{v}\left[\frac{\omega_{i}+\hat{v}_{\alpha i}}{1+\hat{v}_{\alpha} \cdot \omega}\right] \cdot \partial_{k}\left\{\left(E+\hat{v}_{\alpha} \wedge B\right) f_{\alpha}\right\} d v \frac{d y}{|y-x|}
\end{aligned}
$$

integrated over $|y-x| \leqq t$ and over all $v \in \mathbb{R}^{3}$. The derivative $\partial_{k}$ is broken into $T_{j}$ and $S$ components as before. Each integration by parts in the tangential variables $T_{j}$ brings in a term at the base $t=0$ of the cone. Repeating Theorem 4 of [GS1] with a bit simpler notation, we write the result as

$$
\partial_{k} E^{* i}=A_{z}+A_{w}+A_{T T}+A_{T S}+A_{S T}+A_{S S} .
$$

The various terms are given as follows. (For notational simplicity, we drop the subscripts $\alpha$ and take $m_{\alpha}=e_{\alpha}=1$.)

$$
\begin{aligned}
A_{T T} & =\iint a(\omega, \hat{v}) f d v \frac{d y}{|y-x|^{3}}, \\
A_{T S}+A_{S T} & =\iint b(\omega, \hat{v}) S f d v \frac{d y}{|y-x|^{2}}=\iint \nabla_{v} b(\omega, \hat{v}) \cdot(E+\hat{v} \wedge B) f d v \frac{d y}{|y-x|^{2}},
\end{aligned}
$$




$$
A_{\mathrm{SS}}=\iint c(\omega, \hat{v}) S^{2} f d v \frac{d y}{|y-x|}
$$

where the kernels $a, b$ and $c$ are given explicitly in [GS1] and the integration ranges over $|y-x| \leqq t$. The expression $A_{z}$ is the sum of all the terms involving the initial data, namely,

$$
A_{z}=\partial_{k} E_{z}^{* i}+\frac{1}{t^{2}} \iint_{|y-x|=t} d(\omega, \hat{v}) f_{0}(y, v) d v d S_{y}+\frac{1}{t} \int_{|y-x|=t} e(\omega, \hat{v}) S f(0, y, v) d v d S_{y},
$$

where $d$ and $e$ are kernels which once again are bounded for bounded $v$. These integrals come from the passage from $E_{T}^{*}$ to $A_{T T}$, and from $E_{S}^{*}$ to $A_{S T}$, respectively. However in the passage from $E_{T}^{*}$ to $A_{T T}$ we also pick up the term

$$
A_{w}=-\int d(\omega, \hat{v}) f(t, x, v) d v,
$$

because of the singularity $|y-x|^{-2}$ at the vertex of the cone where $y=x$. Now we shall estimate each term in (34).

The "vertex term" is estimated using Lemma 4 as

$$
\left|A_{w}\right| \leqq c \int f(t, x, v) d v \leqq c \varepsilon_{0}(1+t)^{-3},
$$

which is more than sufficient. The "base term" $A_{z}$ has several parts. One is $\partial_{k} \mathscr{E}^{i}$, which is an integral involving derivatives of $E_{0}$ and $B_{0}$ up to order two, obtained by differentiating (29). Since the integration is on the sphere $|y-x|=t$ and since $|y| \leqq k$, it has support in $|t-| x|| \leqq k$. Also, it is of the order $O\left(t^{-1}\right)$. Hence it is $O\left((t+|x|+2 k)^{-1}(t-|x|+2 k)^{-2}\right)$. The second part of $A_{z}$ is the derivative of the second term in $E_{z}^{*}$ given in (29). It is $O\left(t^{-1}\right)$ times an integral over $|y-x|=t$, and so is estimated in the same way as the first part. The integrals appearing in (35) have the same general form. In the last term in (35) appears the expression $S f(0, x, v)=-\left(E_{0}+\hat{v} \wedge B_{0}\right) \cdot \nabla_{v} f_{0}$, which once again has its support in $|x| \leqq k$. Therefore

$$
\left|A_{z}(t, x)\right| \leqq c(t+|x|+2 k)^{-1}(t-|x|+2 k)^{-2}\left(\left\|E_{0}\right\|_{C^{2}}+\left\|B_{0}\right\|_{C^{2}}+\left\|f_{0}\right\|_{C^{1}}\right) .
$$

The kernel $a(\omega, \hat{v})$ in $A_{T T}$ is bounded because $|v| \leqq \beta$. We break $A_{T T}$ up into two parts. The part over the "base" or "shell" $1 \leqq|y-x| \leqq t$ is less than

$$
\begin{aligned}
c & \int_{1 \leqq|y-x| \leqq t} \int f(t-|x-y|, y, v) d v \frac{d y}{|y-x|^{3}} \\
& \leqq c\left\|f_{0}\right\|_{0} \int_{1 \leqq|y-x| \leqq t}(t-|x-y|+|y|+2 k)^{-3} \frac{d y}{|y-x|^{3}} \\
& =\frac{c \varepsilon_{0}}{|x|} \int_{0}^{t-1} \int_{a}^{b} \frac{\lambda d \lambda}{(\tau+\lambda+2 k)^{3}} \frac{d \tau}{(t-\tau)^{2}},
\end{aligned}
$$

where $a=|r-t+\tau|, b=r+t-\tau$ and $r=|x|$ as before, by Lemmas 4 and 7 . The last integral is estimated as follows. (Assume $t>2$ or else the estimate is trivial.) It is broken into the intervals $[0, t / 2]$ and $[t / 2, t-1]$ : 


$$
\begin{aligned}
\int_{0}^{t / 2} & \leqq \frac{4}{t^{2}} \int_{0}^{t / 2} \int_{a}^{b} \frac{d \lambda}{(\lambda+\tau+2 k)^{2}} d \tau \leqq \frac{4}{t^{2}} \int_{0}^{t} \frac{b-a}{(a+\tau+2 k)(b+\tau+2 k)} d \tau \\
& =\frac{4}{t^{2}(t+r+2 k)}\left\{\int_{0}^{(t-r)_{+}} \frac{2 r}{t-r+2 k} d \tau+\int_{(t-r)_{+}}^{t} \frac{2(t-\tau)}{2 \tau+r-t+2 k} d \tau\right\} \\
& \leqq \frac{4}{t^{2}(t+r+2 k)}\left\{2 r+\frac{1}{t-r+2 k} \int_{(t-r)_{+}}^{t} 2(t-\tau) d \tau\right\} \\
& \leqq c(t+r+2 k)^{-2}(t-r+2 k)^{-1} r,
\end{aligned}
$$

since $r \leqq t+k$. On the other hand,

$$
\begin{aligned}
\int_{t / 2}^{t-1} & \leqq(t / 2+2 k)^{-3} \int_{t / 2}^{t-1} \int_{a}^{b} \lambda d \lambda \frac{d \tau}{(t-\tau)^{2}}=4 r(t / 2+2 k)^{-3} \int_{t / 2}^{t-1} \frac{d \tau}{t-\tau} \\
& \leqq c r(t+1)^{-3}(1+\log (t / 2)) \leqq \frac{c r \log (t+r+2 k)}{(t+r+2 k)^{3}}
\end{aligned}
$$

since $r \leqq t+k$. This takes care of the "base" part of $A_{T T}$.

Because of the singularity at $y=x$, we must use the fact that the kernel $a(\omega, \hat{v})$ has zero average on $|\omega|=1$. Therefore the "tip" of $A_{T T}$, that is, the integral over $|y-x|<1$, is

$$
\int_{|y-x|<1} \int a(\omega, \hat{v})[f(t-|x-y|, y, v)-f(t-|x-y|, x, v)] d v \frac{d y}{|y-x|^{3}} .
$$

We use the Mean Value Theorem to represent the difference, and then use Lemma 5. We note that the integration ranges only over the set

$$
\mathscr{A}(\tau)=\{v:|X(0, \tau, z, v)| \leqq k \text { for all } z \in[x, y]\},
$$

where $[x, y]$ is the line segment joining $x$ and $y$ and where $\tau=t-|x-y|$. By Lemma 3 , this set has diameter at most $c /(\tau+2)$. Therefore the integral is bounded by

$$
c\left\|f_{0}\right\|_{1} \int_{|y-x|<1}(2+t-|x-y|)^{-3} \frac{d y}{|y-x|^{2}} \leqq \frac{c \varepsilon_{0}}{(1+t)^{3}},
$$

which is more than sufficient for our purposes. This completes the estimation of $A_{T T}$.

Next, $A_{T S}+A_{S T}$ is bounded by

$$
c\left\|f_{0}\right\|_{0}\|K\|_{0} \frac{1}{r} \int_{0}^{t} \int_{a}^{b}(\tau+\lambda+2 k)^{-4}(\tau-\lambda+2 k)^{-1} \lambda \varphi d \lambda \frac{d \tau}{t-\tau},
$$

where $\varphi=0$ for $\lambda>\tau+k$, as we did several times before by the definition of $\|K\|_{0}$ and by Lemmas 4 and 7 . To estimate this integral, we note that $(\tau-\lambda+$ $2 k)^{-1} \varphi \leqq k^{-1}$ and $\tau+\lambda+2 k \geqq \tau+a+2 k \geqq t-r+2 k$. So the integral is less than

$$
\frac{1}{k r(t-r+2 k)} \int_{0}^{t} \int_{a}^{b} \frac{\lambda d \lambda}{(\tau+\lambda+2 k)^{3}} \frac{d \tau}{t-\tau} \leqq c(t-r+2 k)^{-2}(t+r+2 k)^{-1}
$$

by the previous estimate of the integral in (30). 
It remains to estimate $A_{S S}$. To do this, we decompose it into five terms following Eq. (66) of [GS1] as

$$
4 \pi A_{S S}=\mathrm{I}+\mathrm{II}+\mathrm{III}+\mathrm{IV}^{\prime}+\mathrm{IV}^{\prime \prime}
$$

The first is

$$
\begin{aligned}
|\mathrm{I}| & =\left|\iint f(E+\hat{v} \wedge B) \cdot \nabla_{v}\left[\nabla_{v} c \cdot(E+\hat{v} \wedge B)\right] \frac{d v d y}{|y-x|}\right| \\
& \leqq c \iint f d v|K|^{2} \frac{d y}{|y-x|} \\
& \leqq c\left\|f_{0}\right\|_{0}\|K\|_{0}^{2} \frac{1}{r} \int_{0}^{t} \int_{a}^{b} \frac{\lambda d \lambda d \tau}{(\tau+\lambda+2 k)^{5}(\tau-\lambda+2 k)^{2}}
\end{aligned}
$$

by Lemmas 2, 4 and 7 again. The second is

$$
\begin{aligned}
|\mathrm{II}| & =\left|\iint f \nabla_{v} c \cdot S(E+\hat{v} \wedge B) \frac{d v d y}{|y-x|}\right| \\
& \leqq c\left\|f_{0}\right\|_{0}\|K\|_{1} \frac{1}{r} \int_{0}^{t} \int_{a}^{b} \frac{\log (\tau+\lambda+2 k) \lambda d \lambda}{(\tau+\lambda+2 k)^{4}(\tau-\lambda+2 k)^{2}} .
\end{aligned}
$$

The integral in I is less than the integral in II, so it suffices to estimate the latter. In the integral in II we replace $\log (\tau+\lambda+2 k)$ by $\log (t+r+2 k)$ and $\lambda$ by $\tau+\lambda+2 k$ in order to obtain

$$
\begin{aligned}
& \log (t+r+2 k) \int_{0}^{t} \int_{a}^{b} \frac{d \lambda d \tau}{(\tau+\lambda+2 k)^{3}(\tau-\lambda+2 k)^{2}} \\
& \leqq \frac{1}{2} \log (t+r+2 k) \int_{|t-r|}^{t+r} \frac{d \alpha}{(\alpha+2 k)^{3}} \int_{-k}^{\infty} \frac{d \beta}{(\beta+2 k)^{2}} \\
& =c \log (t+r+2 k)\left\{(|t-r|+2 k)^{-2}-(t+r+2 k)^{-2}\right\} .
\end{aligned}
$$

We replace $|t-r|$ by $t-r$ to obtain at most

$$
\begin{gathered}
4 c r(t+2 k)(t-r+2 k)^{-2}(t+r+2 k)^{-2} \log (t+r+2 k) \\
\leqq 4 c r(t-r+2 k)^{-2}(t+r+2 k)^{-1} \log (t+r+2 k),
\end{gathered}
$$

as desired.

The third term in $A_{S S}$ is

$$
\mathrm{III}=\iint \hat{c}_{j k} f \partial_{j} K^{k} \frac{d v d y}{|y-x|}
$$

where $\hat{c}_{j k}=\left(\delta_{j k}-\hat{v}_{j} \hat{v}_{k}\right)\left(1+|v|^{2}\right)^{-1 / 2} c(\omega, \hat{v})$ is bounded. Hence III is estimated exactly as II was. Next, the fourth term

$$
\left|\mathrm{IV}^{\prime}\right|=\left|-\iint \hat{c}_{j k} K^{k} \frac{\omega_{j}}{1+\hat{v} \cdot \omega} \nabla_{v} \cdot(f K) \frac{d v d y}{|y-x|}\right| \leqq c \iint f d v|K|^{2} \frac{d y}{|y-x|}
$$


is estimated exactly as I was. The fifth term is

$$
\mathrm{IV}^{\prime \prime}=\iint \hat{c}_{j k} K^{k}\left[\delta_{j l}-\frac{\omega_{j} \hat{v}_{l}}{1+\hat{v} \cdot \omega}\right] T_{l} f \frac{d v d y}{|y-x|} .
$$

As above, the integration ranges over the ball $|y-x| \leqq t$. We integrate by parts in $T_{l}$, obtaining on the one hand the term on $|y-x|=t$,

$$
\frac{1}{t^{2}} \int_{|y-x|=t} \hat{c}_{j k} \omega_{j}(1+\hat{v} \cdot \omega)^{-1}\left(E_{0}+\hat{v} \wedge B_{0}\right) f_{0} d v d S_{y},
$$

which is treated just like the integrals in $A_{z}$. On the other hand, we also obtain terms bounded by

$$
c \iint_{|y-x| \leqq t} f\left|\nabla_{x} K\right| \frac{d v d y}{|y-x|}+c \iint_{|y-x| \leqq t} f|K| \frac{d v d y}{|y-x|^{2}} .
$$

Of these resulting integrals, the first one is estimated just as II was, and the second one is estimated just as $A_{T S}+A_{S T}$ was. This completes the proof of Lemma 8.

\section{The Non-Relativistic Model}

The same techniques provide global solutions of the non-relativistic VlasovMaxwell system (VM). The system VM is the same as RVM except that $\hat{v}_{\alpha}$ is replaced by $v / m_{\alpha}$. The analogue of Theorem 1 is the following, where $c$ is the speed of light.

Theorem 4. For each $k>0$ and $c>l>0$, there exist constants $\varepsilon_{0}>0$ and $c>\beta>0$ such that if $f_{\alpha 0}(x, v)$ are non-negative $C^{1}$ functions with supports in $\{|x| \leqq k,|v| \leqq l\}$, if $E_{0}(x)$ and $B_{0}(x)$ are $C^{2}$ functions with supports in $\{|x| \leqq k\}$ which satisfy the required constraints (5), and if the data satisfy (6), then there exists a unique $C^{1}$ solution of VM for all $t, x, v$ with the given initial data such that (7) holds.

Since the details are almost identical to those of Theorem 1, we sketch the proofs of Lemmas 1 and 2 only. For simplicity we drop the subscript $\alpha$ and take $c=1, m_{\alpha}=1$. Now $l<1$, so we have $P(0)<1$, where $P(t)$ is defined in Sect. 3 (the supremum is taken over $|x| \leqq k,|v| \leqq l$ there). On the support of $f(t, x, v)$, then, $|X(s, t, x, v)| \leqq k+s P(t)$, so that

$$
s-|X(s, t, x, v)|+2 k \geqq k+(1-P(t)) s \geqq(1-P(t))(k+s),
$$

which is the analogue of Lemma 1 . As in Lemma 2, we can write

$$
\begin{aligned}
|V(0, t, x, v)-v| & \leqq \int_{0}^{t}|E(s, X(s))+V(s) \wedge B(s, X(s))| d s \\
& \leqq\|K\|_{0}(1+P(t)) \int_{0}^{t}(k+s)^{-2}(1-P(t))^{-1} d s \\
& \leqq k^{-1}\|K\|_{0}(1+P(t))(1-P(t))^{-1},
\end{aligned}
$$

which leads to the inequality

$$
P(t) \leqq \ell+k^{-1}\|K\|_{0}(1+P(t))(1-P(t))^{-1} .
$$


Since the function $x \rightarrow(1+x)(1-x)^{-1}$ is positive for $0 \leqq x<1$, we conclude that if $\ell<\beta<1$ and $\|K\|_{0}$ is sufficiently small (depending on $\ell, k$ and $\beta$ ), then $P(t)<\beta$ for all $t$.

\section{References}

[BD] Bardos, C., Degond, P.: Global existence for the Vlasov-Poisson equation in 3 space variables with small initial data. Ann. Inst. Henri Poincaré, Analyse non linéaire 2, 101-118 (1985)

[BDH] Bardos, C., Degond, P., Ha, T.-N.: Existence globale des solutions des équations de Vlasov-Poisson relativistes en dimension 3. C. R. Acad. Sci. Paris 301, 265-268 (1985)

[GS1] Glassey, R., Strauss, W.: Singularity formation in a collisionless plasma could occur only at high velocities. Arch. Ration. Mech. Anal. 92, 59-90 (1986)

[GS2] Glassey, R., Strauss, W.: High velocity particles in a collisionless plasma. Math. Methods Appl. Sci. 9, 46-52 (1987)

[H] Horst, E.: Global solutions of the relativistic Vlasov-Maxwell system of plasma physics. Habilitationsschrift, Universität München 1986

[J] John, F.: Blow-up of solutions of nonlinear wave equations in three space dimensions. Manuscr. Math. 28, 235-268 (1979)

Communicated by C. H. Taubes

Received January 9, 1987; in revised form May 18, 1987 